\title{
Understanding the concept of chemotherapy-related nausea: the patient experience
}

A. MOLASSIOTIS, RN, PHD, University of Manchester, School of Nursing, Midwifery e Social Work, Manchester, UK, C.T. STRICKER, PHD, MSN, CRNP, AOCN, Abramson Cancer Center of the University of Pennsylvania, Philadelphia, PA, B. EABY, MSN, CRNP, OCN, Abramson Cancer Center of the University of Pennsylvania, Philadelphia, PA, L. VELDERS, BA, Abramson Cancer Center of the University of Pennsylvania, Philadelphia, PA, USA, \& P.A. COVENTRY, PHD, University of Manchester, School of Nursing, Midwifery et Social Work, Manchester, UK

MOLASSIOTIS A., STRICKER C.T., EABY B., VELDERS L. \& COVENTRY P.A. (2008) European Journal of Cancer Care 17, 444-453

Understanding the concept of chemotherapy-related nausea: the patient experience

The aim of this study was to explore the experience of chemotherapy-related nausea in patients with cancer. A qualitative study was carried out with 17 patients who had experienced nausea during their chemotherapy in the UK and USA. Nausea was described as distressing and complex symptom. Patients attempted to construct an understanding of nausea using cognitive processes such as analysing their experience of nausea and related symptoms, attributing causation to nausea and comparing their experiences not only to their own expectations, but also to others' symptom experiences. A number of concurrent and associated symptoms linked with nausea were identified. Preliminary evidence emerged for nausea as part of a cluster of symptoms. Anti-emetic medication, provider-directed management strategies and self-management strategies were used by patients to minimize the effects of nausea. Self-management techniques, such as dietary strategies, were rooted in participants' understanding of nausea and their beliefs about what caused nausea, and there was little evidence of guidance from professionals beyond advice about medication management. This study reveals some of the complexities behind chemotherapy-induced nausea, including a potential symptom cluster, and contributes towards a clearer understanding of this symptom and its effects on patients' lives.

Keywords: nausea, concept, chemotherapy, cancer, experiences, symptom cluster, quality of life.

Significant developments in anti-emetic therapy over the past two decades have improved the control of chemotherapy-related vomiting. By contrast, chemotherapy-related nausea, both acute and delayed, is still a significant problem in clinical practice, with $42-52 \%$ of patients experiencing nausea on any one day in routine practice (Glaus et al. 2004), and daily rates greater

Correspondence address: Alexander Molassiotis, Professor of Cancer \& Supportive Care, University of Manchester, School of Nursing, Coupland III, Coupland Street, Manchester M13 9PL, UK (e-mail: alex.molassiotis@ manchester.ac.uk).

Accepted 22 August 2007

DOI: $10.1111 / j .1365-2354.2007 .00872 . x$

European Journal of Cancer Care, 2008, 17, 444-453 than $60 \%$ in high risk populations such as women with breast cancer receiving anthracycline-based chemotherapy (Stricker \& Velders 2005). Surprisingly, despite improvements in the management of vomiting, postchemotherapy nausea seems to have been increased (Roscoe et al. 2000). Furthermore, clinicians often underestimate the experience of nausea, especially with regards to delayed nausea (Grunberg et al. 2004; Liau et al. 2005).

Chemotherapy-induced nausea and vomiting can have a profound effect on the cancer treatment experience (Bergkvist \& Wengstrom 2006) and is associated with negative effects on daily life and overall quality of life, including effects on food intake, weight loss, effects on social interactions, dehydration, difficulty with sleeping 
and anxiety (Foubert \& Vaessen 2005; Bergkvist \& Wengstrom 2006). In a qualitative study of patients' experiences, unmanaged nausea was constant in some patients and made them exhausted for long after chemotherapy, making recovery between cycles longer (Bergkvist \& Wengstrom 2006). The impact of nausea is greater than that of vomiting (Griffin et al. 1996) and nausea has proven to be more difficult to control.

Anti-emetic trials have traditionally focused primarily on vomiting and emetic episodes, upon which the effectiveness of many anti-emetic drugs is judged. Little attention has been directed to the concept of chemotherapyinduced nausea despite the fact that it is increasingly recognized that nausea and vomiting are related but separate entities (ASHP 1999; Miller \& Kearney 2004). The need for these two symptoms to be treated as two separate entities is also advocated (Miller \& Kearney 2004). Some reasons for the suboptimal management of chemotherapyinduced nausea may be the subjectivity of the symptom, lack of a focus on nausea assessment using validated tools, a limited understanding of the pathophysiology of nausea, patients failing to report their nausea experience, and clinicians' failure to appreciate the impact of nausea in patients' lives.

Besides descriptive work focusing on the frequency and severity of chemotherapy-related nausea in conjunction with vomiting, there has been little work directed at a better understanding of nausea in patients receiving chemotherapy. Hence, the aim of this study was to describe the experience of chemotherapy-induced nausea in patients' own words, as a first step in understanding this symptom more clearly, with the ultimate goal of improving nausea assessment and management. The specific objectives of the study were:

- to conceptualise nausea after chemotherapy and understand the symptom experience;

- to assess the presence of other symptoms linked with nausea;

- to understand the impact of chemotherapy-related nausea on patients' lives; and

- to explore the techniques used by patients to manage chemotherapy-related nausea.

\section{METHODS}

This study was a qualitative descriptive study that collected data through one-time interviews with participants. Although no particular qualitative paradigm was followed, the study was directed towards understanding and exploring the lived experience of the participants in order to develop a richer and deeper understanding of a human phenomenon (in our case being nausea) (Van Manen 1990). Phenomenological approaches to data collection were broadly applied, drawing on specific a priori topics and using probing, verification of interpretations and the responses to interview questions to contribute thematically to knowledge production. Patients were recruited from two comprehensive oncology centres, one in the UK and one in the USA. Patients were invited to take part in the study if they had received chemotherapy over the past 4 months and experienced nausea in any of the previous four cycles of chemotherapy. A research assistant approached patients who met inclusion criteria, all of whom had previously completed a nausea-vomiting scale during their chemotherapy as part of a larger study (Molassiotis et al. 2007). The study was explained to them, and patients consented to participate in an interview about their nausea experience. Criteria for participation included age 18 years or older, receiving any type of outpatient chemotherapy with high or low emetogenic potential, ability to communicate in English, and willingness to participate in the study. The study was approved by the Ethics Committee or Institutional Review Board of each hospital involved.

Interviews lasted for 20-40 min and were carried out either face-to-face at one of the patients' follow-up visits (USA interviews) or over the telephone (UK and USA interviews). An interview guide was developed, and questions comprised the following five main themes: (1) defining nausea in the participant's own words; (2) a description of the nausea experience for each individual; (3) how much of a problem it had been and how it affected their lives; (4) any other sensations or symptoms they experienced together with nausea; and (5) how participants attempted to manage this symptom themselves. All interviews were tape-recorded with the patients' permission and were transcribed verbatim. Data collection ended at each centre when no new information was obtained from the interviews (data saturation).

\section{DATA ANALYSIS}

Content analysis was used to analyse the interview data, utilizing a system of open coding where data were analysed, compared and categorized (Strauss \& Corbin 1990). The five questions used as the interview guide became broad themes around which data were organized, and responses related to each one of these themes were grouped together. The interview data were selfcommunicating with minimal need for further explanation and interpretation. The themed responses formed a clear story. Within each broad theme, codes and sub-codes 
were developed. Codes and sub-codes were developed independently by both the UK and USA teams and then were compared with ascertain agreement. Agreement was high between the coders, with only minor disagreement in the codes. The disagreement was about where an item (participant quotation) fit better when the quotation appeared to fit in more than one theme. This was resolved by repeating such quotations under more than one theme. Data were analysed using the qualitative package Atlas-ti Version 5.2.

\section{RESULTS}

\section{Sample}

The sample consisted of 17 participants out of 21 that were approached for inclusion in the study; nine from the USA centre and eight from the UK centre. The four patients who refused to participate did not give a reason for this. The participants' mean age was 60.5 years $(\mathrm{SD}=11.9$, range $24-74)$. There were 10 male and seven female participants and their diagnoses included lung cancer $(n=9,53 \%)$, lymphoma $(n=2,11.7 \%)$, sarcoma $(n=2,11.7 \%)$, breast cancer $(n=2,11.7 \%)$, head and neck cancer $(n=1,6 \%)$ and genitourinary cancer $(n=1,6 \%)$. The chemotherapy drugs administered were cisplatin $(n=8,47 \%)$ and doxorubicin $(n=4,23.4 \%)$, followed by cisplatin with concurrent gemcitabine $(n=2,11.7 \%)$, ABVD (doxorubicin, bleomycin, vincristine, dacarbazine) or DHAP (cisplatin, cytarabine, dexamethasone) protocols $(n=1$ each, $6 \%)$, and gemcitabine $(n=1,6 \%)$, representing both highly emetogenic and lower emetogenic chemotherapy protocols.

\section{Definition of nausea}

Participants identified nausea as being a difficult symptom to describe. They often described nausea in terms of a physical sensation that was localized to a particular part of the body. Two specific categories of nausea definitions emerged: (1) feeling the need to vomit; and (2) sensations (such as an ache, queasiness, unsettled feelings localized to the stomach, and in one participant, also in the chest). Participants described these sensations in their own words, such as 'shake up in the stomach', 'upset stomach', 'queasiness', and being 'ill in the stomach'.

Well ... A sense of my stomach feeling unsettled and that I might bring up something that [is] there, even if there isn't anything there.... it's basically, it's the feeling that the contents of what's in the stomach don't want to stay there, that's what it feels like. It feels like it wants to leave. (US, 10:40,70)
It's like a complete ache over your stomach and your chest cavity and it feels like a washing machine inside. It doesn't make you feel... it annoys you more than anything. Stops you doing things. (UK, 5:3) A feeling of queasiness and uneasiness in your stomach. (US, 9:5)

\section{Nausea experience}

A key theme emerged when participants were asked to describe their personal experience of nausea. Participants consistently described their attempts to construct an understanding of nausea based on their own experiences. Participants used cognitive processes to analyse their experience of nausea and related symptoms and to attribute causation to nausea, and they reflexively compared their experiences not only to their own personal expectations, but also to others' symptom experiences. Participants used these expectations on an ongoing basis to help them adapt to and cope with nausea to varying degrees, and modified their expectations over time based on their own experiences (Fig. 1). The quotes below illustrate the above sub-themes:

I more or less knew what to expect the second time ... I had an idea of what was going to happen. The first time I was totally lost. (US, 10:19)

... I just tried to grin and bear it as I knew it was only going to be for a couple of days. I mean, I've known it is a maximum of four days after treatment and then I'm back to my normal self ... (UK 5:12)

... you kind of accept it, you forget it. (UK 1:25)

Many similar quotes suggest that patients were acclimating to nausea.

Patients attempted to analyse the nausea experience to make sense of it or attributed causes to their chemotherapy-induced nausea and vomiting (CINV) experience, sometimes causes unrelated to their chemotherapy.

... I was sick and I couldn't work out what was making me sick... (US 3:12)

...but I don't think it came from this [chemotherapy], it's just that I didn't feel so good... (US $10: 481$

... the nausea didn't start until about day four ... and now today, it started yesterday so I assume it's going to go away by day four... I got a nasty taste in my mouth [making him nauseous] but you get that with chemotherapy. That's just accepted I think. (UK 1:7, 24) 


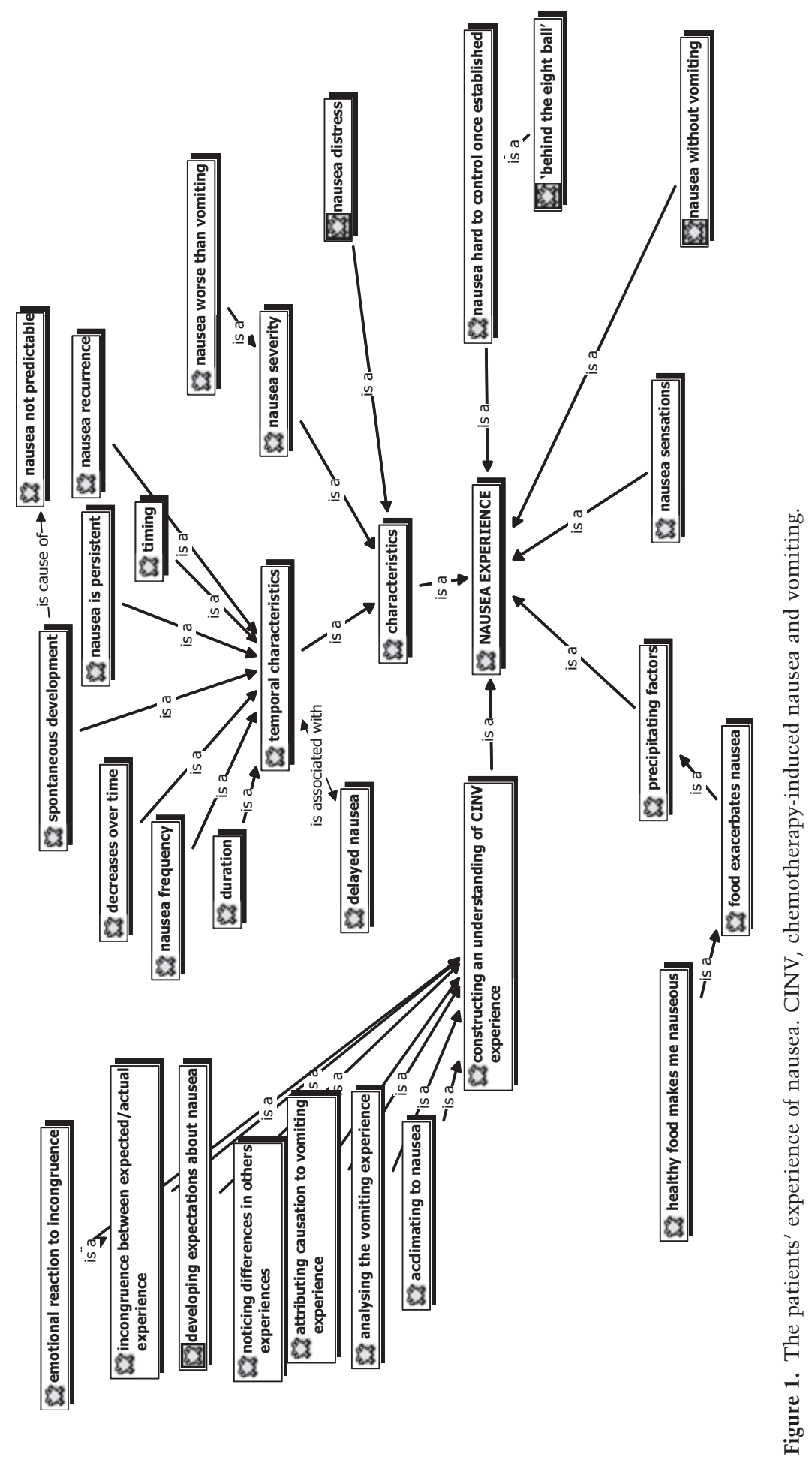


Part of the process of understanding CINV was to compare their experiences with those of others and notice differences in other patients receiving the same chemotherapy. As one patient illustrates:

... I've heard of people who have been through the whole course, six odd weeks and they've had no loss, of erm, energy, just can't understand it. Some people must be built like. . . Makes you wonder what's going wrong. I've always looked after myself ... (US 9:20)

Participants at times described incongruence between what they expected to happen and their actual experience. A few participants described emotional reactions to this incongruence, as depicted in the following quotes:

It's gone back to how it was on cycle one, I'm suffering... (UK 1:1)

But I've just come to the end of the full treatment now - three weeks ago - and I was a bit disappointed my appetite was very poor. And, as I say, the nausea is still [there] even though I take the dom ... [domperidone] whatever they are called. (US 2:5)

Patients talked about the characteristics that shape their nausea experience (Fig. 1). Participants often described their nausea experience in terms of temporal characteristics. They described nausea in terms of frequency, duration, timing with respect to chemotherapy and predictability. Duration was variable between participants, ranging from a day or so to weeks after the end of chemotherapy, and from very infrequent to constant. Patients with greater duration and frequency of nausea also noted greater distress and described more effects on their daily life. They spoke about spontaneous and unpredictable development of nausea that distressed them a lot, and also about persistent or recurrent nausea. Many did not experience vomiting.

I started [having nausea] Saturday, I had chemo Wednesday, I don't know which day it was, but Saturday, Sunday and Monday I had really extreme nausea that we just couldn't get under control. There was no vomiting. (US 10:60)

Well, on the first couple of times I felt very, very nauseous and wanting to retch. By the fourth treatment, I was feeling much ... you know, a lot better. But there's always been an underlying, slight nausea ... But every now and again it pops back again. (UK $7: 131$

Patients communicated that nausea was worse than vomiting, as after vomiting they usually felt better.
I'd rather be sick than have nausea ... to be honest I'd rather have the sickness because you can do something about it ... (UK, 5:6, 16)

Patients felt that nausea was difficult to control once established.

Somehow I got behind the eight ball and never [small laugh] never caught up or even go ahead. So I was pretty bad for three days and then got better. (US 10:61)

The longer the duration and the more severe the nausea was, the more distressed patients were.

... but it's [nausea] a most, most unpleasant thing and I could do without it. (US 7:23)

Another dose of the first one [chemotherapy] would have finished me I think... I only had three treatments. The first one nearly killed me and I was no-man's land for a week and a half. Never felt so sick in all my life. (UK, 8:5, 1)

Participants also described precipitating factors, with the most common being food. Often food, even the thought of food, would exacerbate patients nausea.

... the first couple of times ... I couldn't look at stuff [food]. I did keep trying, that was pretty horrible. I was very nauseous then. (UK 7:14)

I don't eat the right things because the only things that stay down are crappy things, like healthy food makes me nauseous. (US 10:80)

Patients also described many sensations they felt while being nauseous, as described earlier under the definition of nausea. It is worth noting that a couple of patients did experience nausea but this neither bothered them nor affected their daily life in any way. Their nausea level, however, was mild.

\section{Concurrent and associated symptoms}

A range of symptoms linked with nausea were described by the patients. These were categorized as concurrent (those that were not consistently present along with nausea) and associated symptoms (those symptoms that participants clearly described as temporally occurring in conjunction with nausea). The number of quotations corresponding to a particular symptom is listed after each symptom. Concurrent symptoms included sleep disturbance $(n=4)$ and fatigue $(n=7)$, bloating $(n=2$, which was a different sensation from nausea), sore throat $(n=2$, occurred especially in those that also vomited), sweating $(n=2)$, weakness $(n=4)$, dizziness $(n=1)$ and headache 
$(n=2)$, flu-like symptoms $(n=3)$ and feeling hot and cold $(n=2)$. Combinations of these symptoms were also present when nausea developed.

It was a weird feeling, it's almost like I was feeling I was coming down with the flu or something... [I felt] kind of washed out during the time I was nauseous. (US 10:14, 22)

Well, flu-like symptoms as well, and half the time you've got headache, feeling hot and cold. (UK, 8:7)

I get very tired but whether that's got anything to do with it I don't know. Very tired, occasionally, I can drop off to sleep. (UK, 7:10).

Sweats, yes. Tiredness, yes. You see nausea covers a lot, a spectrum. (US 9:6)

Associated symptoms that participants described as clearly occurring in conjunction with nausea included burping $(n=1)$ associated with a hot sensation $(n=1)$, a 'feeling [of needing] to swallow, push things down' $(n=1)$, and regurgitation $(n=1)$; intolerance of smells $(n=1)$; taste disturbance $(n=7)$; loss of appetite $(n=15)$; vomiting $(n=11)$; dry heaves $(n=4)$. As was expected, vomiting was frequently, although not consistently, reported to occur in association with nausea. Frequently associated symptoms emerged, including loss of appetite, vomiting and taste changes, and they often occurred simultaneously, suggesting a possible symptom cluster.

Yes, poor appetite, and another thing ... my taste has gone strange... I can taste, but it doesn't taste like food should taste ... it is really weird. (UK 7:8,9)

It affects your appetite, it affects your taste and [inaudible] ... it's been six weeks now and I'm only just starting to eat food properly. (UK, 8:9)

Queasiness, sometimes sweating, intolerance to smells. (US 10:6)

\section{Effects on daily life}

The majority of patients in the study mentioned that nausea interfered with their daily life, although it was clear that the effects of nausea depended on its severity. Those participants who had mild nausea reported little, if any, interference. Those with more severe nausea reported negative effects on their activities of daily living, social life (i.e. not going out for a meal with friends) and quality of life, and described having emotional responses to nausea (i.e. feeling irritable, not wanted to be bothered). The effect on eating was the most consistently reported by participants. On some occasions, it was not the nausea per se that affected their social life, but rather associated symptoms, such as loss of appetite/no enjoyment of food or fatigue. Often it was not clear, in the presence of many associated symptoms, which one(s) affected daily life the most, suggesting complex interrelationships between the concurrent and associated symptoms of nausea. Some participants noted a decrease in their physical activity, with the most extreme example of being unable to get out of bed. Sleep disturbance was also present in several patients, and at times was clearly linked to experiencing nausea. One patient referred to feeling completely 'blacked out'. The following quotes illustrate some of the above effects:

You don't want to be bothered, you don't want to eat, but it's something you've got to do ... so I tried to control myself and didn't get mad when people were talking to me I don't want to go anywhere or do anything.... I basically blacked out for almost a week, where I could hardly, where barely could get out of bed. I mean literally like with, you know, to the bathroom all I could do. I had to call the neighbour to let the dog out. (US 10:17, 24, 74)

It's very weird, it's horrible when you can't enjoy your food, because I normally enjoy my food. (UK 7:17)

It affects your appetite, if affects your taste... it's been six weeks now and I'm only just starting to eat food properly. You eat because you have to. (UK 8:9)

... [nausea] is a horrible thing to have, especially when you're trying to go to sleep. (UK, 5:4).

A couple of participants felt that social support from their relatives moderated the effects of nausea in their lives.

[going out:] the wife is always with me so I am lucky this way ... I do take them [anti-emetic tablets], my wife insists. (US 9:13,19)

Some participants also discussed that other symptoms (i.e. fatigue, sleep disturbance, pain) were more severe and, despite the moderating affect of social support, they were more debilitating than nausea itself. A few participants also noted that nausea did not affect their life at all, but these were individuals with only mild nausea.

\section{Management of nausea}

Three major categories were developed in relation to the management of nausea: provider-directed management, taking medication and self-management techniques.

Provider-directed management was mainly centred on anti-emetic medication management. When one antiemetic regimen did not work well, this was changed later 
to a different regimen. One patient (PT075) reported that her doctor reduced her doses of chemotherapy in subsequent cycles as a result of severe CINV.

[husband talking] ... there has been a marked difference since she [the patient] started taking steroids, there's no question about that, and in terms of appetite. (UK 7:15)

We have some different medication this time. (USA 10:651

Compared with providers, participants used a more diverse array of strategies to minimize nausea and its effects, although medication was still the most common strategy for managing nausea (anti-emetics). Sixteen of 17 subjects mentioned medication as a strategy for managing nausea. While anti-emetics were helpful for many of the participants, they were often not fully effective and some participants expressed little confidence in their ability to help, particularly participants who experienced more severe levels of nausea. Participants developed expectations about nausea after the first cycle that appeared to help them better prepare for subsequent cycles:

The second time [second cycle] I knew, I had an idea of what was going to happen ... The first time I was totally lost. I guess I [am] able to handle it better knowing what was going to happen and where it was going to happen. (US 10:18, 19, 20)

Patients felt that prevention is critical and took their anti-emetic tablets on time, without waiting for nausea to develop. Some patients also bought other medication (i.e. antacids) over the counter to help them soothe their stomach.

Self-management approaches were common. Besides acceptance of and adjustment to the previously experienced pattern of chemotherapy-related nausea (acclimating to nausea), patients used a variety of techniques to manage nausea. Some patients were able to prepare better for and cope with symptoms of nausea by relating their past experiences of nausea to their ongoing symptoms. In this sense, patients' cognitive responses to their nausea triggered a behavioural response, leading to improved selfregulation of their symptoms. This approach is in keeping with the self-regulatory model of illness developed by Leventhal (Leventhal et al. 1984). In this model, patients' cognitive representations about their illness are regarded as key determinants of how patients cope with and critically appraise their symptoms. The self-regulatory model has been used in a number of chronic health states, including cancer, to explain patient responses to symptoms and treatments (Buick 1997).
One of the key behavioural responses to nausea was dietary self-management. Dietary strategies were the most common non-pharmacological self-care approach, and participants reported modifying their diets $(n=5)$, increasing fluid intake $(n=2)$, forcing themselves to eat $(n=3)$, avoiding eating when nauseous $(n=3)$ and avoiding acidic food/drink $(n=1)$.

I ate, but I didn't want to really look at food. (US, 10:131

I am eating but I've got to eat very soft foods, and I couldn't eat a big Sunday dinner. (UK, 2:5)

Participants also frequently reported modifying their activities and resting in order to cope with nausea. Participants frequently used distraction techniques $(n=7)$ and rest $(n=5$; i.e. sitting or laying down, napping or 'taking it easy') to manage nausea. A couple of participants used art and painting as a distraction, one US subject strongly believed in the psychology of the mind to overcome nausea, and yet another participant used prayer.

I am back to my drawing and painting ... this painting and drawing I am doing now it is a therapy and it takes your mind off it [nausea] which helps. (UK 9:11, 16)

I am just using psychology and so forth ... the power of the mind in the theory is very strong I just don't think about it. I block it out, think of something else ... Say your prayers. Ask God and he will take it away. (US 10:37, 38, 86, 88)

Well ... I'm not working a the moment and I notice it [nausea] quite a lot. But if I was busy, or shopping or I was working then I wouldn't have felt it as much I don't think. (UK 5:7).

Just taking something [medication] and not doing an awful lot you know, and taking it easy - once you get over that first week it's ok you know. (UK 8:12)

Sleeping more, eating less ... (UK 1:16)

\section{DISCUSSION}

To our knowledge, this is the first qualitative study to examine exclusively the experience of chemotherapyinduced nausea. It identified that nausea is not only a difficult symptom to describe, but also a complex symptom with which participants struggled to cope by reflexively using experiential data. Nausea often affected participants' quality of life and daily living. Participants provided rich descriptions of the nausea experience, associated and concurrent symptoms, management strategies, and the effect of nausea in their lives. 
Nausea was described using a number of physical sensations, most confined in the stomach area. Participants described nausea as a complex symptom, and hence the definition health professionals often use in practice of 'feeling sick' appears to be an oversimplification. The concurrent symptoms and sensations described earlier add to the understanding of what nausea is, as they are suggestive of autonomic system involvement (i.e. sweats, or feeling hot and cold).

The associated symptoms are also of interest, and preliminary evidence emerged in support of a symptom cluster consisting of nausea, loss of appetite, taste disturbance, vomiting and possibly intolerance of smells. Causal directionality cannot be established from the present qualitative study. Our qualitative data are reflective of findings from an earlier quantitative study that used factor analysis in 220 elderly patients with lung cancer, and found that the symptoms of fatigue, nausea, appetite loss, weight loss, taste changes and vomiting form a cluster (Gift et al. 2004). Understanding clusters of symptoms can assist patients in being better prepared for the symptom experience and health professionals in planning more effective interventions that target more than one symptom concurrently (Miaskowski et al. 2004). Future research on clusters of symptoms should focus in further clarifying particular symptom clusters and the synergistic effects among the symptoms of a cluster on patient morbidity. It should also test innovative interventions that could affect not only individual symptoms but the whole cluster of symptoms.

Participants tried to construct an understanding of their nausea experience. Many felt this was inevitable and expressed their resignation to bearing this symptom, but others worked to identify a predictable pattern by analysing their experience. Frustration and other emotional reactions were evident when expectations did not meet with actual experiences. Patient preparation and education about the symptom experience could help patients in making sense of this experience and be prepared for what to expect. The effectiveness of such approaches has been demonstrated in several prior studies (Hagopian 1996; Christman \& Cain 2004).

Nausea affected many aspects of participants' lives, especially those related to eating and feeling weak, consistent with prior research (Foubert \& Vaessen 2005; Bergkvist \& Wengstrom 2006). Severe nausea prevented participants from carrying on as normal. Nutritional intake appeared to decrease significantly during chemotherapy in nauseated patients, an effect which could potentially prolong individuals' recovery between cycles (Bergkvist \& Wengstrom 2006). Some patients felt nau- seous even weeks after the end of treatment, with significant effects in their quality of life.

Likely due to the patient-centred interview approach used in this study, healthcare provider interventions were described to a limited degree, and focused solely on medication management. In contrast, participants described in detail the self-management techniques they used in an attempt to minimize nausea and its effects on their lives. Quite striking was the lack of direction that participants received from their providers regarding selfmanagement strategies beyond use of anti-emetic medication. In spite of this apparent lack of guidance and education, participants reported diverse self-care strategies. Dietary strategies were common. Dietary modification might improve the nausea-loss of appetite-taste changes group of symptoms, although our information on this area is largely anecdotal (Molassiotis \& Borjeson 2006). This is an important area of self-management research in the future. For example, avoiding foods with strong smells, not drinking fluids half an hour before any meal, avoiding fried or fatty foods, drinking plenty of water and having small meals more frequently could be simple dietary approaches to help decrease nausea (Molassiotis \& Borjeson 2006). Participants also described using distraction techniques to manage nausea. Limited prior research has documented the potential of distraction techniques in reducing symptom distress, although not specific to chemotherapy-related nausea. For example, virtual reality techniques as a distraction method have been shown to significantly decrease symptom distress in a crossover trial of 20 women receiving chemotherapy (Schneider et al. 2004). Other potential methods to decrease nausea include acupressure (Dibble et al. 2000; Molassiotis et al. 2007), hypnosis, or herbs, such as ginger or peppermint, that have been used to sooth an upset stomach. However, these methods need further evaluation.

Oncology providers have a critical role to play in preparing patients receiving chemotherapy for the experience of nausea and helping them to understand that there are many different options for the management of nausea. Health professionals should be vigilant in assessing this complex and distressing symptom, and in offering support to patients. Participants in this study clearly used self-management strategies in an attempt to manage their nausea. However, patients received very little guidance from healthcare providers about what might be useful beyond medication management. Health providers (physicians, nurses and/or nutritionists) should educate patients about self-care strategies for managing the potential cluster of symptoms related to nausea, and 
provide tailored educational interventions towards helping patients to use such strategies more effectively. This should be the focus of future research, as evidence is this area is scarce. Self-management behaviours could be taught to patients before chemotherapy; one such educational intervention, using audiotapes, led to a significant decrease in the symptoms of nausea, vomiting, fatigue and taste changes in a randomised trial of 70 women receiving their first cycle of chemotherapy (Williams \& Schreier 2004). Furthermore, informational audiotapes were effective in teaching patients self-care practices in another trial of 75 patients receiving radiation therapy (Hagopian 1996).

Several anti-emetic medications, non-pharmacological interventions, and self-management techniques exist, but it is unlikely that any single approach will be completely effective for all patients. The challenge is to learn how to incorporate newer anti-emetics and nonpharmacological methods in an individualized way into existing anti-emetic regimens, especially in high-risk patients. Increasing our understanding of the biological nature of nausea could lead in the future in the development of more potent anti-nausea medication. Chemotherapy-related nausea is a complex symptom, potentially part of a cluster of symptoms, and innovative approaches to its management are necessary. Self-care strategies are important and often used by patients in managing their symptom experience, and there is an urgent need to develop the evidence base of such approaches and educate patients about the most optimal self-care strategies that they can utilize when they are away from the hospital setting.

\section{ACKNOWLEDGEMENTS}

This study was supported by an unrestricted grant from Merck \& Co.

\section{REFERENCES}

ASHP. (1999) Therapeutic guidelines on the pharmacologic management of nausea and vomiting in adult and pediatric patients receiving chemotherapy or radiation therapy or undergoing surgery. American Journal of Health-System Pharmacy 56, 729-764.

Bergkvist K. \& Wengstrom Y. (2006) Symptom experiences during chemotherapy treatment - with focus on nausea and vomiting. European Journal of Oncology Nursing 10, 21-29.

Buick D.L. (1997) Illness representations and breast cancer. coping with radiation and chemotherapy. In: Perceptions and of Health and Illness (eds Petrie K.J. \& Weinman J.), pp. 379-410. Harwood Academic, Amsterdam, the Netherlands.
Christman N.J. \& Cain L.B. (2004) The effects of concrete objective information and relaxation on maintaining usual activity during radiation therapy. Oncology Nursing Forum 31, E39E45.

Dibble S.L., Chapman J., Mack K.A. \& Shih A.S. (2000) Acupressure for nausea: results of a pilot study. Oncology Nursing Forum 27, 41-47.

Foubert J. \& Vaessen G. (2005) Nausea: the neglected symptom? European Journal of Oncology Nursing 9, 21-32.

Gift A.G., Jablonski A., Stommel M. \& Given C.W. (2004) Symptom clusters in elderly patients with lung cancer. Oncology Nursing Forum 31, 203-212.

Glaus A., Knipping C., Morant R., Böhme C., Lebert B., Glawogger B., Fernandez Ortega P., Hüsler A. \& Deuson R. (2004) Chemotherapy-induced nausea and vomiting in routine practice: a European perspective. Supportive Care in Cancer 12, 708-715.

Griffin A.M., Butow P.N., Coates A.S., Childs A.M., Ellis P.M., Dunn S.M. \& Tattersall M.H. (1996) On the receiving end. V: patient perceptions of the side effects of cancer chemotherapy in 1993. Annals of Oncology 7, 189-195.

Grunberg S.M., Deuson R.R., Mavros P., Geling O., Hansen M., Cruciani G., Daniele B., De Pouvourville G., Rubenstein E.B. \& Daugaard G. (2004) Incidence of chemotherapy-induced nausea and emesis after modern antiemetics. Cancer 100, 2261-2268.

Hagopian G.A. (1996) The effects of informational audiotapes on knowledge and self-care behaviours of patients undergoing radiation therapy. Oncology Nursing Forum 23, 697-700.

Leventhal H., Nerenz D.R. \& Steel D.J. (1984) Illness representations and coping with health threats. In: A Handbook of Psychology and Health, Vol. 4 (eds Baum A. \& Singer J.), pp. 219-252. Erlbaum, Hillsdale, NJ, USA.

Liau C.T., Chu N.M., Liu H.E., Deuson R. \& Chen J.S. (2005) Incidence of chemotherapy-induced nausea and vomiting in Taiwan: physicians' and nurses' estimation vs. patients' reported outcomes. Supportive Care in Cancer 13, 277-286.

Miaskowski C., Dodd M. \& Lee K. (2004) Symptom clusters: the new frontier in symptom management research. Journal of the National Cancer Institute. Monographs 32, 17-21.

Miller M. \& Kearney N. (2004) Chemotherapy-related nausea and vomiting-past reflections, present practice and future management. European Journal of Cancer Care 13, 71-81.

Molassiotis A. \& Borjeson S. (2006) Nausea and vomiting. In: Nursing Patients with Cancer: Principles and Practice (eds Kearney N. \& Richardson A.), pp. 415-438. Elsevier, Oxford, UK.

Molassiotis A., Helin A.M., Dabbour R. \& Hummerston S. (2007) The effects of P6 acupressure in the prophylaxis of chemotherapy-related nausea and vomiting in breast cancer patients. Complementary Therapies in Medicine 15, 3-12.

Molassiotis A., Coventry P.A., Stricker C.T., Clements C., Eaby B., Velders L., Rittenberg C. \& Gralla R.J. (2007) Validation and psychometric assessment of a short clinical scale to measure chemotherapy-induced nausea and vomiting: the MASCC Antiemesis Tool (MAT). Journal of Pain and Symptom Management 34, 148-159.

Roscoe J.A., Morrow G.R., Hickok J.T. \& Stern R.M. (2000) Nausea and vomiting remain a significant clinical problem: trends over time in controlling chemotherapy-induced nausea and vomiting in 1413 patients treated in the community. Journal of Pain and Symptom Management 20, 113-121.

Schneider S.M., Prince-Paul M., Allen M.J., Silverman P. \& Talaba D. (2004) Virtual reality as a distraction intervention for women receiving chemotherapy. Oncology Nursing Forum 31, 81-88. 
Strauss A.L. \& Corbin J. (1990) Basics of Qualitative Research. Sage, Thousand Oaks, CA, USA.

Stricker C.T. \& Velders L. (2005) Predictors of chemotherapyinduced nausea and vomiting in women with early stage breast cancer [abstract]. Supportive Care in Cancer 13, 422.

Van Manen M. (1990) Researching Lived Experience: Human
Science for an Action Sensitive Pedagogy. University Of New York Press, New York, NY, USA.

Williams S.A. \& Schreier A.M. (2004) The effect of education in managing side effects in women receiving chemotherapy for the treatment of breast cancer. Oncology Nursing Forum 31, E16-E23. 